\title{
Supermicrosurgical reconstruction of knee defect using superior medial genicular perforator as a recipient vessel
}

\begin{abstract}
Hoon Kim
Department of Plastic and Reconstructive Surgery, International St. Mary's Hospital, Catholic Kwandong University College of Medicine, Incheon 404834, South Korea.
\end{abstract}

Address for correspondence: Dr. Hoon Kim, Department of Plastic and Reconstructive Surgery, International St. Mary's Hospital, Catholic Kwandong University College of Medicine, 25, 100 Gil, Simgok-Ro, Seo-Gu, Incheon 404834, South Korea.

E-mail: prshoonkim@gmail.com

\begin{abstract}
A 24-year-old male presented after being involved in a motorcycle accident and was found to have soft tissue defects of the knee with exposure of patella. Due to the severe injury from the popliteal fossa to the posterior aspects of the lower leg, repair with a free flap from anterolateral thigh perforator was planned instead of local calf muscle flap. Preoperative angiography was performed, and it showed that superior medial genicular perforator was patent compared with unreliable filling of the superior lateral genicular perforator. The soft tissue defect was repaired using the superior medial genicular perforator as the recipient vessel. This was performed by creating perforator to perforator anastomosis (supermicrosurgery). The flap survived successfully, and the patient was able to ambulate in a few weeks without serious complications. This case indicates that superior medial genicular perforator can be used as the recipient vessel for covering the soft tissue defects of the knee caused by blunt injury.
\end{abstract}

Key words:

Soft tissue defect of the knee, superior medial genicular perforator, supermicrosurgery

\section{INTRODUCTION}

Soft tissue defect of the knee remains challenging and problematic to reconstructive surgeons. Prerequisite for the reconstruction of this region include the flexibility, durability and thickness of the skin paddle to sustain the motion of the knee joint. Numerous surgical trials have been performed using musculocutaneous flaps, fasciocutaneous flaps, perforator flaps, and free flaps to repair these defects with varying degrees of success. ${ }^{[1-3]}$

Local flaps created from the calf muscle are preferred primary surgical option. However, local flap may not be an

\begin{tabular}{|l|l|}
\hline \multicolumn{2}{|c|}{ Access this article online } \\
\hline Quick Response Code: & Website: \\
\hline & www.parjournal.net \\
\cline { 2 - 2 } & \\
\hline & \\
\hline
\end{tabular}

option when there is injury to the donor site endangering the vascularity. Although the free flaps are less affected in terms of the donor site selection, selection of the recipient vessel in the vicinity of knee defect remains problematic.

Since perforator to perforator anastomosis (supermicrosurgery) emerged, this technique has been vastly used for reconstructive surgeries without regard to the recipient vessel. We report a case using the superior medial genicular perforator as the recipient vessel and supermicrosurgery techniques to cover the soft tissue defect of the knee.

\section{CASE REPORT}

A 24-year-old male was injured in a motorcycle accident. Patient had a knee injury with soft tissue defect measuring $12 \mathrm{~cm} \times 7 \mathrm{~cm}$ and exposure of the patella was noted [Figure 1]. Physical examination revealed severe contusion of the posterior calf. Because of these findings, repair using local gastrocnemius 


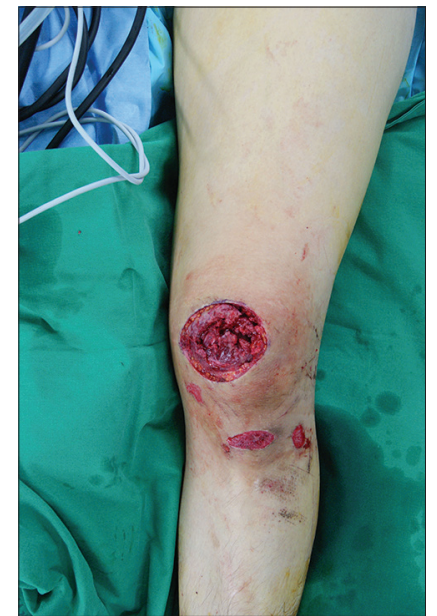

Figure 1: Preoperative finding shows soft tissue defect of the left knee measuring $12 \mathrm{~cm} \times 7 \mathrm{~cm}$ with patella exposure

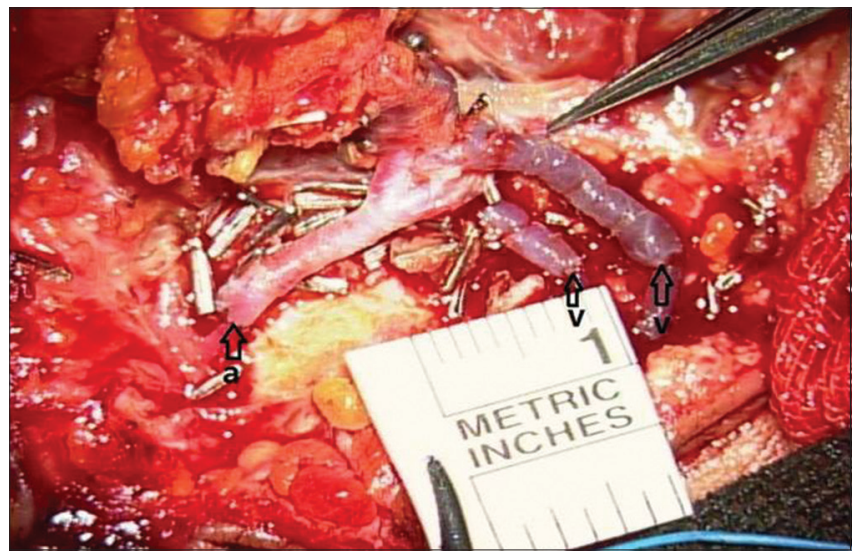

Figure 3: Supermicrosurgical anastomosis of one artery $(0.6 \mathrm{~mm})$ and two veins $(0.4 \mathrm{~mm}, 0.7 \mathrm{~mm})$ with $10-0$ and $11-0$ nylon was made in an end-to-end fashion (a: artery, v: vein)

musculocutaneous flap was excluded to avoid unreliable vascularity of the donor site. Instead, a free flap reconstruction using a vessel in the vicinity of the knee as the recipient was planned.

Conventional angiography instead of computed tomographic angiography was performed to predict the direction of vascular flow around the traumatized knee. It showed abrupt cutoff with retrograde filling of the superior lateral genicular perforator compared with intact superior medial genicular perforator [Figure 2]. Using the intraoperative hand-held Doppler, perforator of superior medial genicular artery was targeted and identified. Elevation of anterolateral thigh perforator free flap with $3 \mathrm{~cm}$ pedicle length was performed and the superior medial genicular perforator was identified under a microscope. Perforator to perforator anastomoses of one artery $(0.6 \mathrm{~mm}$, descending branch of lateral circumflex femoral artery with superior medial genicular artery) and two veins $(0.4 \mathrm{~mm}$ and $0.7 \mathrm{~mm}$, venae comitantes) with 10-0 and 11-0 nylon were made in an end-to-end fashion [Figure 3]. Donor site was closed with meshed allogeneic dermal matrix, followed by split thickness skin graft $(0.3048 \mathrm{~mm})$.

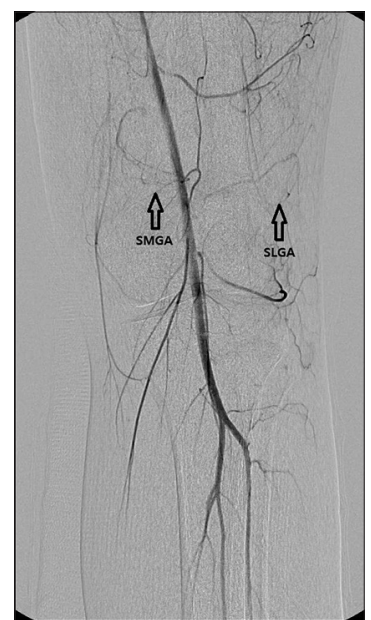

Figure 2: Conventional angiography shows the abrupt cutoff with retrograde filling of the superior lateral genicular perforator compared with intact superior medial genicular perforator (SMGA: superior medial genicular artery, SLGA: superior lateral genicular artery)

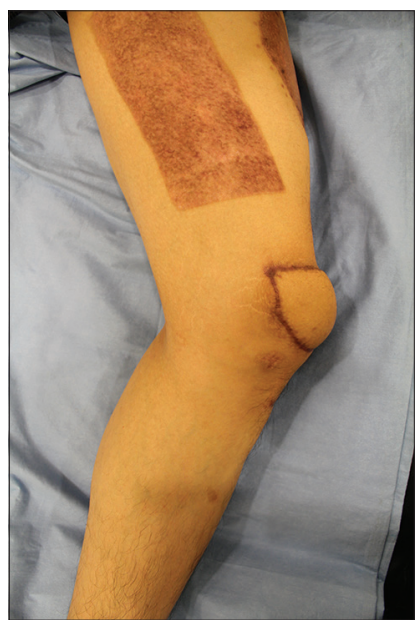

Figure 4: Acceptable functional and aesthetic appearance was obtained at postoperative month 11

The flap survived successfully, and the patient had functional ambulation within 15 days after surgery without any complications. Full flexion of the knee joint was achieved by postoperative week 4 . Patient was also able to squat without any discomfort. The patient was satisfied with the contour of the flap at postoperative month 11 [Figure 4].

\section{DISCUSSION}

Reconstruction of soft tissue defects surrounding the knee has been well-known for its difficulty and strenuous nature of the process. Damage to the soft tissue around the knee can be caused by trauma, cancer resection, and the exposure of prosthesis.

Several musculocutaneous flaps including gastrocnemius, sartorius, vastus medialis, and vastus lateralis flaps have been applied successfully to cover the soft tissue defect of the knee. Other fasciocutaneous flaps, island flaps and perforator flaps based on the sural artery, superior lateral genicular artery, and the reverse flow of 
descending branch of lateral femoral circumflex artery have also been used. ${ }^{[4-6]}$ However, bulky contour of the flap, functional impairment of the donor site, and discomfort on ambulation due to the scar extended from the donor site to the knee may be encountered with these flaps.

To avoid these drawbacks, several free flaps have been introduced. ${ }^{[7-9]}$ However, free flaps using the source vessels may threaten the vascularity around the traumatized knee joint. The evolution of microsurgical techniques has allowed surgeons to anastomose vessels between perforators that are smaller than $0.8 \mathrm{~mm}$ in caliber. These techniques are termed as supermicrosurgery. Hong and Koshima used this refined technique on 25 soft tissue defects over knee joint and minimized donor site morbidity. ${ }^{[9]}$ However, their promising results are extremely dependent on the expertise of the surgeon. They preferred free style reconstruction without identifying the recipient vessels, while they could easily find the recipient vessels in the plane between the fascia and muscle.

In this report, we demonstrate the use of conventional angiography to identify superior medial genicular perforator vessel as the recipient in this patient. To the best of our knowledge, this is the first case of supermicrosurgical reconstruction using superior medial genicular perforator as a recipient vessel. Although this procedure is technically demanding, the use of conventional angiography to identify the recipient vessels made it less tasking. Compared to conventional local flap modalities, this technique creates less scarring, providing better contour of the knee and decreased discomfort on ambulation. The patient could flex the knee joint fully and perform exercises such as squats without any discomfort in four weeks.
Further investigation with larger cases should be performed for validation. Nevertheless, this case implies the use of supermicrosurgical techniques and superior medial genicular perforator as an alternative to repair soft tissue defect surrounding the knee when the conservative local flap technique may not be reliable.

\section{REFERENCES}

I. Zheng HP, Lin J, Zhuang YH, Zhang FH. Convenient coverage of soft-tissue defects around the knee by the pedicled vastus medialis perforator flap. J Plast Reconstr Aesthet Surg 2012;65: I I 5 I-7.

2. Liu TY, Jeng SF, Yang JC, Shih HS, Chen CC, Hsieh CH. Reconstruction of the skin defect of the knee using a reverse anterolateral thigh island flap: cases report. Ann Plast Surg 2010;64:198-20I.

3. Fujiwara T, Chen CC, Ghetu N, Jeng SF, Kuo YR. Antegrade anterolateral thigh perforator flap advancement for soft-tissue reconstruction of the knee: case report. Microsurgery 2010;30:549-52

4. Heo C, Eun S, Bae R, Minn K. Distally based anterolateral-thigh (ALT) flap with the aid of multidetector computed tomography.J Plast Reconstr Aesthet Surg 2010;63:e465-8.

5. Dai J, Chai Y,Wang C,Wen G. Proximal-based saphenous neurocutaneous flaps: a novel tool for reconstructive surgery in the proximal lower leg and knee.J Reconstr Microsurg 201 3;29:373-8.

6. Wiedner $\mathrm{M}$, Koch $\mathrm{H}$, Scharnagl $\mathrm{E}$. The superior lateral genicular artery flap for soft-tissue reconstruction around the knee: clinical experience and review of the literature. Ann Plast Surg 201 I;66:388-92.

7. Kim JS, Lee HS, Jang PY, Choi TH, Lee KS, Kim NG. Use of the descending branch of lateral circumflex femoral artery as a recipient pedicle for coverage of a knee defect with free flap: anatomical and clinical study. Microsurgery 2010;30:32-6.

8. Fang T, Zhang EW, Lineaweaver WC, Zhang F. Recipient vessels in the free flap reconstruction around the knee. Ann Plast Surg 2013;71:429-33.

9. Hong JP, Koshima I. Using perforators as recipient vessels (supermicrosurgery) for free flap reconstruction of the knee region. Ann Plast Surg 2010;64:291-3.

How to cite this article: Kim $\mathrm{H}$. Supermicrosurgical reconstruction of knee defect using superior medial genicular perforator as a recipient vessel. Plast Aesthet Res 2014;1:81-3.

Source of Support: Nil, Conflict of Interest: None declared.

Received: 11-05-2014; Accepted: 15-07-2014 